\title{
Lung disease in patients with systemic lupus erythematosus
}

\author{
Authors: Tijana Stanojković ${ }^{1}$,Branislava Glisic ${ }^{2}$ \\ ${ }^{1}$ Faculty of Medicine, Military Medical Academy, Belgrade, Serbia \\ ${ }^{2}$ Clinic for Rheumatology, Military Medical Academy, Belgrade, Serbia \\ DOI: https://doi.org/10.26800/LV-142-supp5-38
}

\section{Introduction:}

Sistemic lupus erythematosus is an autoimmune disease, which can affect a lot of organs, including lungs.

\section{The aim:}

The aim of this study was to investigate the frequency of lung involvement in patients with Systemic Lupus Erythematosus (SLE) treated at the Military Medical Academy. Material and methods: 75 patients $(68 ; 90.6 \%$ female) were included in this cross-sectional study. They all met the revised EULAR/ACR classification criteria for SLE. The mean age was $45 \pm 12$ (2278) years. The disease lasted an average of $11 \pm 10(1-38)$ years. In all patients, the presence of symptoms and signs of lung disease, radiographic examination of the lungs, spirometry and carbon $\begin{array}{llll}\text { monoxide factor tung } & \text { (DLCO). }\end{array}$

\section{Results:}

At the time of the trial, two patients had clinically manifest lung disease. Also, one had changes in the lung radiography (bilateral fibrosis). Normal spirometry was reported by $67(89,37 \%)$ patients, ventilation disturbances of restriction type light grade $3(4 \%)$, moderate-severe degree $4(5,3 \%)$ and severe degree $1(1.33 \%)$. Normal DLCO had $19(25,33 \%)$, decreased mild grade $35(46,67 \%)$, moderately-severe $17(22,67 \%)$ and severe grade $4(5,33 \%)$ patients. None of the patients with normal spirometry and reduced DLCO had any clinical or radiographic signs of lung disease.

\section{Conclusion:}

Our study showed that a significant number of patients without clinically and radiographically manifest lung disease and with normal spirographic findings, had a reduced DLCO. Based on these results, we believe that patients with SLE without respiratory symptoms should be monitored pulmonary function (DLCO) periodically for possible development of interstitial lung disease with subsequent pulmonary hypertension.

Keywords: systemic lupus erythematosus; lungs; spirometry; carbon monoxide lung factor transfer 